\title{
Neurological applications
}

for myocardial MIIBG scintigraphy

Cascini Giuseppe Lucio', Cuccurullo Vincenzo²,

Restuccia Antonio'1, Tamburrini Oscar¹, Rotondo Antonio², Mansi Luigi

${ }^{1}$ Department of Radiological Sciences - University of Catanzaro, Catanzaro, Italy

2Department of Radiological Sciences - Second University of

Naples, Naples, Italy

\section{[Received 26 IX 2012; Accepted 28 XI 2012]}

\section{Abstract}

Signs or symptoms of impaired autonomic regulation of circulation are typically present in patients affected by Parkinson's disease (PD), in agreement with the cardiac sympathetic denervation discovered by Goldstein more than 15 yrs. ago. In particular, the majority of PD patients have a diffuse left ventricular myocardial sympathetic denervation, being a normal neurological condition present only in a small number of affected subjects. Actually MIBG cardiac imaging is a universally accepted method to estimate cardiac sympathetic innervations. This review covers the role of MIBG cardiac imaging in PD as well as in other parkinsonisms, focusing the attention on technical problems and pathophysiological premises for cardiac denervation. In particular new emerging data support the role of MIBG as biomarker of $\mathrm{PD}$, also before motor symptoms became clinically evident. Therefore the timing of cardiac noradrenergic denervation in PD is a key issue and we want to update the analysis of autonomic cardiovascular abnormalities studied with MIBG in PD and related disorders.

KEY words: sympathetic innervation, Parkinson disease, parkinsonism, MIBG, myocardium

Nuclear Med Rev 2013; 16, 1: 35-41

Correspondence to: Prof. Luigi Mansi

Medicina Nucleare, Seconda Università di Napoli

P.zza Miraglia, 2-80138 Napoli, Italy

E-mail: luigi.mansi@unina2.it

\section{MIBG and the role of sympathetic cardiac innervations}

Cardiac sympathetic innervation is responsible for hemodynamic adaptations of the cardiovascular system to changing demands. Nor-epinephrine (NE), neurotransmitter of the sympathetic system, is synthesized from the aminoacid tyrosine that is stored in high concentration in synaptic vesicles.

When a stimulus occurs, vesicles containing NE are released into the synaptic space, and NE bind to post synaptic beta-1, beta-2, and alfa receptors, enhancing adenyl-cyclase activity through an intermediary $\mathrm{G}$ protein, finally resulting in the cardiac stimulatory effects.

$\mathrm{NE}$ is taken back into the presynaptic terminal by a protein-mediated sodium energy and temperature-dependent transporter for storage or catabolic disposal that terminates the sympathetic response; this specific process of action is known as "uptake-1 pathway". Moreover part of NE into the synaptic space, is taken up by non-neuronal postsynaptic cells, probably through a passive diffusion (uptake-2 pathway) [1, 2].

Guanethidine, a false neurotransmitter analog of NE, may be chemically modified in meta-lodo Benzyl Guanidine ( $m \mid B G$ ) that can be labeled with radioactive iodine ( ${ }^{123}$ lodine most commonly), becoming ${ }^{123}$ - $m$ IBG. $m$ IBG is actively taken up through the uptake-1 pathway by the postganglionic pre synaptic nerves endings of the adrenergic nervous system. In contrast to NE, ${ }^{123}$ - $-m \mid B G$ is not catabolized by monoamine oxidase or catechol-O-methyltransferase; therefore ${ }^{123} \mathrm{I}-\mathrm{mIBG}$ is retained and localized in myocardial sympathetic nerve endings at a sufficient concentration to be imaged with a conventional gamma camera. On the basis of this pathophysiological premise, ${ }^{123}$ |-MIBG myocardial scintigraphy can non-invasively assess the postganglionic presynaptic cardiac sympathetic nerve endings.

\section{Technical considerations for cardiac MIBG imaging}

${ }^{123}$ I-mIBG injection is performed at rest and requires only a minimal preparation. Many medications, such as antidepressants, antipsychotics, and some calcium channel blockers, potentially interfere with catecholamine uptake and should be stopped at least 
24 hours before radiotracer's injection. A list of medications interfering with MIBG uptake is available at:

www.eanm.org/publications/guidelines/gloncomibg.pdf

After intravenous injection of ${ }^{123} \mathrm{I}-\mathrm{mIBG}$, planar and single photon emission computed tomography (SPECT) images of the chest are obtained using a gamma camera at 15 min (early imaging phase) and 3/4 hrs post-injection (delayed imaging phase).

Cardiac MIBG uptake in the early imaging phase mainly reflects the density of the presynaptic cardiac sympathetic nerve endings, whereas the delayed imaging phase reflects the presynaptic functional tone of the cardiac sympathetic nerve as well. The uptake can be semi-quantitatively evaluated by calculating the heart-to mediastinum $(\mathrm{H} / \mathrm{M})$ ratio by setting regions of interest over the heart and the upper mediastinum chosen on the anterior planar view of the chest as background.

The appropriate dosage of ${ }^{123}$ - $-m \mid B G$ has not yet been definitively established. In several published studies investigators have proposed a dose of $3-5 \mathrm{mCi}$, and this is generally satisfactory for a standard planar image analysis. Nevertheless, because it is often difficult to obtain satisfactory SPECT images using this dosage, particularly in patients with severe cardiac dysfunction or with severe neurological impairment, higher doses allowing a reduced time of acquisition and/or a better count rate may be appropriate.

Although some investigators suggest that only the delayed image should be used for interpretation and analysis because it represents actual neuronal uptake (as opposed to interstitial uptake in the early images), studies from other authors have reported that the estimation of $m \mathrm{IBG}$ washout rate (WR) between early and delayed images may provide additional information. In non-collaborative patients the early and delayed planar acquisitions with the estimation of WR may be considered a reasonable approach. This assumption is particularly true in patients with neurological impairment where motion artifacts may significantly affect image quality in SPECT, even more if myocardial uptake is reduced. In fact, global cardiac uptake as well as the estimation of the heart/mediastinal ratio $(\mathrm{H} / \mathrm{M})$ can be reliably assessed from planar images although the superposition of liver and lungs presenting high ${ }^{123}$ |-mIBG concentrations negatively affects scintigraphic acquisitions mainly in most seriously affected patients.

In the standard procedure, the $\mathrm{H} / \mathrm{M}$ is calculated on a delayed anterior chest planar image by drawing a region of interest (ROI) including the heart $(\mathrm{H})$ and the other one over the upper mediastinum; the mean counts per pixel in the heart are divided by the mean counts per pixel in the mediastinum. The method proposed by various investigators in defining the cardiac $\mathrm{ROI}$ are different, with some authors using a ROI limited to the myocardial wall, others drawing a ROI around the entire heart, and still others drawing the $\mathrm{ROI}$ around the entire heart, but excluding the cavity. In the neurological setting we prefer to draw the $\mathrm{ROI}$ around the entire heart because in these patients a markedly reduced uptake may be present with a poor myocardial representation not allowing a reliable definition of the myocardial wall; although this method is less accurate and produces a lower $\mathrm{H} / \mathrm{M}$ ratio respect to the one constructed on the myocardial mass, on the contrary it is more reproducible in patients with faint uptake, becoming therefore in our opinion the best approach for the evaluation of myocardial denervation, as early marker of PD and as differential parameter between PD and parkinsonims. In order to standardize the image analysis, the ROls depiction is crucial: we try to use in all patients the same mediastinal ROI , of fixed dimensions. Moreover both ROls are depicted on early images then pasted on delayed ones, being frequently the earlier uptake more favorable to define the ROl's position. The procedure is particularly effective when the patient is repositioned in the same position in further controls. Irrespective of which procedure has been used, normal values for $\mathrm{H} / \mathrm{M}$ range from 1.9 to 2.8 with a mean of approximately $2.2[3,4]$.

The $\mathrm{H} / \mathrm{M}$ ratio reflects receptor density and integrity of pre-synaptic nerve terminals and uptake- 1 function. A high ratio indicates a predominant localization of the tracer in the myocardium that is expected for normal hearts, whereas a decreased ratio indicates a lower myocardial uptake, with an increased activity in extra-cardiac structures: therefore these data are in agreement with a reduced cardiac adrenergic receptor density [5].

The late $\mathrm{H} / \mathrm{M}$ ratio combines information on neuronal function from uptake to release through the storage vesicles at the nerve terminals [6].

\section{Sympathetic system in Parkinson's disease}

Parkinson's disease (PD) is the most common neurodegenerative parkinsonism characterized by the degeneration of both dopaminergic and non-dopaminergic neurons, with neuronal intra-cytoplasmic inclusions known as Lewy bodies. Patients with PD typically present tremor, rigidity and bradykinesia. Furthermore PD is characterized by a cardiac dysautonomia determined, as demonstrated in 1997 by Goldstein using cardiac imaging with 18F-Dopamine, by the loss of at least one mechanism in post-ganglionic noradrenergic nerves [7]. After 15 years and many imaging studies performed with different techniques, the cardiac sympathetic denervation is today worldwide accepted as a PD biomarker [8-11] .

Abnormalities of tau and $\alpha$-synuclein have been described in a variety of neurodegenerative diseases, all showing parkinsonism with symptoms as tremor, bradykinesia, rigidity and gait impairment. Main tauopathies with parkinsonism are progressive supranuclear palsy (PSP) and cortico-basal degeneration (CBD). PSP is a clinical entity that shows supranuclear ophthalmoplegia, dystonia, rigidity of the neck and upper trunk, pseudobulbar palsy, and dementia [12] . CBD was first described in 1968 as cortico-dentato-nigral degeneration with neuronal achromasia [13], which shows late middle age onset, progressive asymmetric rigidity, apraxia, tremor, and cortical and extrapyramidal dysfunction [14]. On the other hand, the main synucleinopathies with parkinsonism are Parkinson's disease (PD) and multiple system atrophy with parkinsonism (MSA-P). PD is the most common cause of parkinsonism; MSA was first introduced in 1969 [15], and MSA-P has been recognized as a certain type of MSA showing predominant parkinsonism, autonomic failure, and poor levodopa-response [16]. The clinical differentiation among PSP, CBD, MSA-P and PD is of decisive importance to pre-define the response to therapy and the respective prognosis. At disease onset, it is particularly difficult to precisely diagnose and distinguish these forms presenting with an akinetic-rigid syndrome. Furthermore PD is characterized by a variety of non-motor manifestations, which sometimes dominate the clinical picture and can precede the movement disorder, even by decades. These include constipation, depression, cognitive dysfunction, anosmia, REM behavior disorder, and orthostatic hypotension. The latter three non-motor 

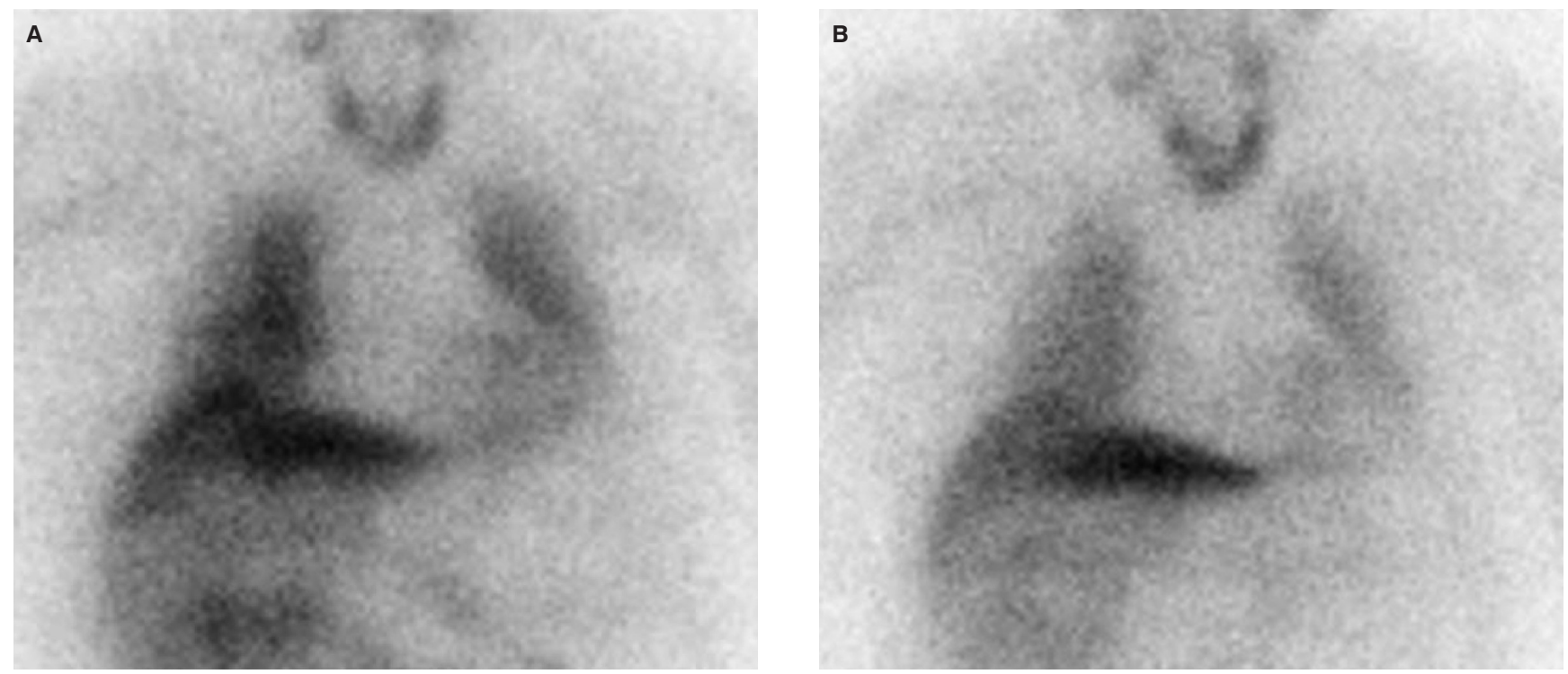

Figure 1. MIBG Cardiac imaging. Early (A) and Delayed (B). The H/M ratios were 1.55 and 1. 45, respectively. Patient was affected by early PD (Y\&H 2)

manifestations are associated with cardiac sympathetic denervation [17-19] (Figure 1A, B).

Almost all patients with PD have at least a partial olfactory dysfunction, and a substantial minority presents anosmia. Among patients with $\alpha$-synuclein pathologies such as PD, the grade of anosmia measured by UPSIT scores, is positively correlated with myocardial noradrenergic innervation as assessed by cardiac sympathetic neuroimaging [19]. REM behavior disorder (RBD) is a new pathological entity associated with PD. RBD patients present an evident myocardial noradrenergic denervation as demonstrated by cardiac sympathetic neuroimaging [21, 22]. Most PD patients may eventually develop dementia. In some patients dementia precedes the movement disorder or dominates the clinical picture, whereas the presence of visual hallucinations and fluctuating cognition leads to a diagnosis of Lewy Body Dementia (DLB) when in presence of insufficient signs in agreement with PD diagnosis. DLB is defined on the basis of a neuroimaging pattern concomitant with an evident cardiac sympathetic denervation. In this regard DLB differs from Alzheimer's disease, in which cardiac sympathetic innervation is generally intact. Because of this distinction, cardiac sympathetic neuroimaging has been proposed as a tool for a differential diagnosis of DLB respect to Alzheimers disease and Frontotemporal Dementia (FTD) [23, 24].

The sympathetic denervation in PD is further responsible for the orthostatic hypotension. This condition when in association with $\mathrm{PD}$, is characterized by a cardiac and extra-cardiac noradrenergic denervation, accompanied by an arterial baroreflex failure, as recently described by Goldstein [25].

The complexity of this scenario is further complicated, from the nuclear medicine point of view, by the ability of MRI to differentiate various pathological entities that coexist in tau and $\alpha$-synuclein abnormalities. Although new MRI techniques, as the thickness measurements of selected areas as well as functional analysis of the answers to specific motor tasks, have been recently introduced with interesting results [26-28], $m I B G$ and dopamine imaging remain an important clinical tool, exclusively providing to the neurologist the functional status of a specifically involved neurotransmission. This scenario will be soon expanded by the availability of hybrid PET-MRI scanners, being also viable when PET and MRI may interact in a more strict connection [29].

\section{The role of cardiac mIBG Imaging in PD}

Being early differentiation of PD respect to other neurodegenerative parkinsonism crucial, there is an important need to improve the diagnostic accuracy of ${ }^{123} \mid$-mIBG myocardial scintigraphy. This radionuclide study was originally developed to assess postganglionic presynaptic cardiac sympathetic nerve endings in heart diseases for pathologies including congestive heart failure, ischemic heart disease, and cardiomyopathy. Subsequently, cardiac uptake was demonstrated to be reduced in patients with Lewy body diseases, such as PD [30, 31] and DLB [32]; furthermore it has been reported to be useful for differentiating PD respect to other parkinsonism as well as DLB respect to Alzheimer's disease (AD). Post mortem studies have shown that the number of cardiac tyrosine hydroxylase (TH)-immunoreactive axons, marker for sympathetic axons, is decreased in pathologically confirmed PD and DLB $[33,34]$, supporting the findings of a reduced cardiac $m I B G$ uptake in Lewy body diseases (Figure 2A, B).

Moreover it has been revealed that PD, DLB and pure autonomic failure (PAF) share a single clinic-pathological entity: Lewy Bodies diseases (LBD) has thus become a general term for these conditions, Lewy bodies having been pathologically observed in the nervous system of patients affected with these disorders [35-38]. LBD present an impairment of adrenergic function and consequently an abnormal myocardial innervation imaging: the involvement of myocardial postganglionic sympathetic nerves may account for the reduction of myocardial $m \mathrm{IBG}$ uptake in patients with $\mathrm{PD}$, as well as in patients with other $\operatorname{LBD}[39,41]$. Also patients with idiopathic RBD usually present a reduction of myocardial mIBG uptake; RBD consists of a wide range of neurodegenerative disorders, but the pathological entity of idiopathic RBD has not yet been confirmed.

In recent papers focusing the attention on diagnostic accuracy of cardiac mIBG for differentiating PD from Essential tremors, 

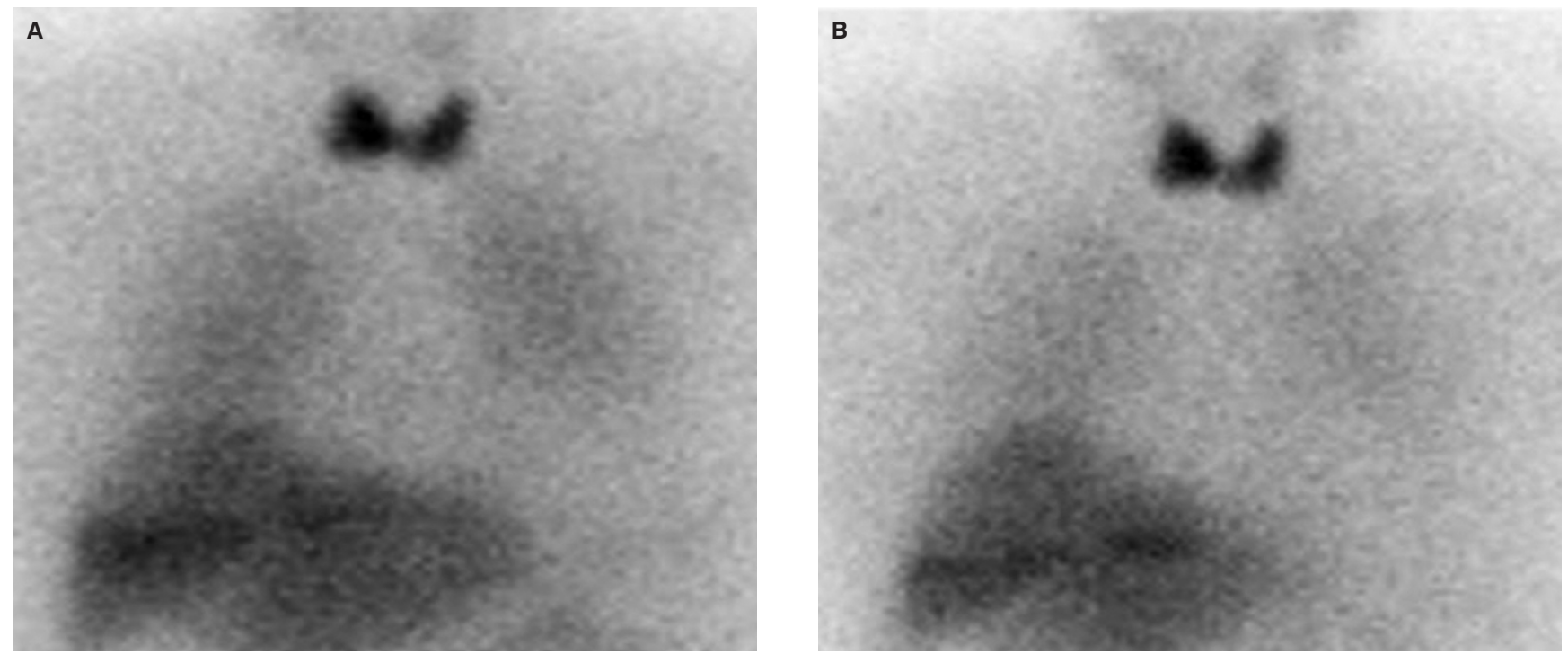

Figure 2. MIBG Cardiac imaging. Early (A) and Delayed (B). The H/M ratios were 1.1 and 1.1. Patient was affected by LBD

as well as PD from other parkinsonism (MSA, PSP and CBD), different methodologies have been adopted, including acquisition parameters, processing, gold standard, and cut-off values. Two meta-analyses performed by Treglia [42] and Orimo [43] have calculated the diagnostic performances of $m I B G$ in PD, MSA, PSP, $\mathrm{CBD}, \mathrm{LBD}$, also considering technical differences.

Analyzing 11 studies published with the aim to distinguish PD from other parkinsonism including MSA, PSP and CBD, Orimo et al. [43] have observed a sensitivity ranging from $64.5 \%$ to $100 \%$ by using delayed $\mathrm{H} / \mathrm{M}$ ratios, while specificities were between $23.1 \%$ and $100.0 \%$. In this meta-analysis, the pooled sensitivity and specificity were respectively $89.7 \%$ (95\% Cl: $81.6 \%, 94.5 \%)$ and $82.6 \%$ (95\% Cl: $60.2 \%, 93.7 \%)$. The $\mathrm{H} / \mathrm{M}$ early ratios in the same group of studies ranged from $67.5 \%$ to $92.0 \%$, while the specificities were between $44.4 \%$ and $100.0 \%$. In the evaluation of only 5 studies including patients with PD at an early stage (Hoehn-Yahr stage 1 or 2) a sensitivity and specificity respectively of $94.1 \%$ and 80.2 has been calculated. Finally Orimo and colleagues have meta-analyzed the accuracy of $m \mathrm{IBG}$ in differentiating PD from MSA and PD from PSP. They have reported a $90 \%$ sensitivity and a $82 \%$ specificity from 10 studies focused on PD against MSA, while in PD versus PSP the sensitivity and specificity were $91,4 \%$ and $78 \%$ respectively. This meta-analysis demonstrated a pooled sensitivity of $89.7 \%$ and a specificity of $82.6 \%$ using the delayed $\mathrm{H} / \mathrm{M}$ ratio for differentiating PD from other neurodegenerative parkinsonisms, supporting the use of the delayed ratio, although similar diagnostic performances have been obtained in this setting applying early $\mathrm{H} / \mathrm{M}$ ratio. Similar mIBG performances were detected in $\mathrm{PD}$ at an early stage; although these data have been obtained at the present in a little series, they are in accordance with the hypothesis of $m I B G$ as marker of pre-motor PD. It has to be pointed out that in PD myocardial $m \mathrm{IBG}$ 's uptake irreversibly and progressively decrease with disease's progression; sometimes the cardiac denervation detected by $m \mathrm{IBG}$ precedes motor symptoms and is irrespective of dopamine loss in the striatum. These evidences enhance the mIBG impact in the clinical scenario of patients with PD.
For differentiating PD from MSA, different meta-analysis [44, 45] reported a sensitivity of $90 \%$ and a specificity of $85 \%$. It has to be remembered that in MSA the central and preganglionic neurons are pathologically involved whereas postganglionic sympathetic neurons are usually spared. Therefore, as reduced cardiac mIBG uptake implies a decreased density of the post-ganglionic presynaptic cardiac sympathetic nerve endings, cardiac mIBG uptake is generally not reduced in MSA. However, a reduction of cardiac mIBG uptake was found in some patients with MSA [46, 47]. Indeed, although the number of tyrosine hydroxylase-immunoreactive (TH-ir) axons of the heart is markedly decreased in PD and basically preserved in MSA [47, 48], 6 out of 15 subjects with longer duration of illness and with pathologically confirmed MSA exhibited a slightly decreased number of TH-ir axons [49]. This result suggests that a reduction of cardiac mIBG uptake can occur in MSA. Nevertheless, mIBG uptake in MSA patients tends to be normal or, when slightly decreased, with values generally higher than in PD (Figure 3A, B).

Similar $m$ IBG performances in differentiating PD from PSP (sensitivity: 91.4\%; specificity: 78\%) have been reported. As patients with PSP usually do not have autonomic dysfunction, thus cardiovascular autonomic dysfunction being an exclusionary feature of PSP, cardiac $m$ IBG uptake should not be reduced in PSP. Conversely, some patients with PSP showed reduced cardiac $\mathrm{mIBG}$ uptake probably due, at least in part, to the effects of tricyclic anti-depressants which may reduce cardiac $m$ IBG uptake, non-suitably suspended before the radionuclide scan. Therefore, careful attention must be given to administered medications and to their suspension, to avoid pitfalls and mistakes

Another recent meta-analysis performed by King [50] has confirmed the utility of $m \mathrm{IBG}$ scintigraphy in discriminating LBD from non-LBD: these authors have identified an ideal $\mathrm{H} / \mathrm{M}$ threshold value $(H / M=1.77)$ that may be useful in differentiating these clusters. This threshold value has been obtained by a ROC analysis performed using $\mathrm{H} / \mathrm{M}$ values of different studies; the $\mathrm{H} / \mathrm{M}$ threshold value of 1.77 yielded $94 \%$ of sensitivity and $91 \%$ of 

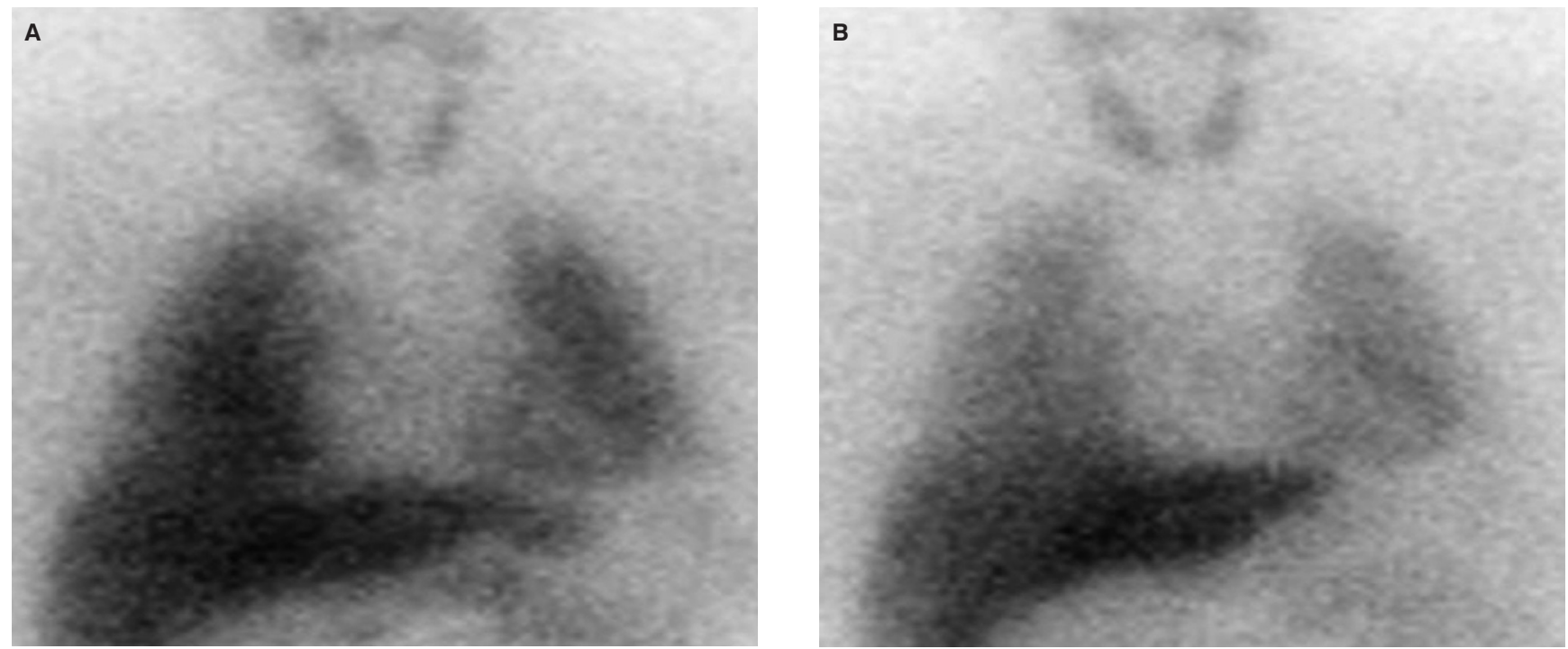

Figure 3. MIBG Cardiac imaging. Early (A) and Delayed (B). The H/M ratios were 2.1 and 2.0. Patient was affected by Primary Idiopathic Freezing of Gait

specificity for the discrimination of LBD from non-LBD. Furthermore in this meta-analysis are reported the mean values of $\mathrm{H} / \mathrm{M}$ ratio in controls (2.19), in PD (1.31), in MSA (2.13), in PSP (2.22), FTD (1.85), DLB (1.25) and RBD (1.32). These data demonstrated that $\mathrm{DLB}, \mathrm{PD}$ and $\mathrm{RBD}$ are clustered around 1.2/1.3 of $\mathrm{H} / \mathrm{M}$ ratio, clearly separated from MSA and PSP.

Another issue that may be addressed by $\mathrm{mIBG}$ is the evaluation of patients with freezing. Freezing is a form of gait disturbance with sudden and transient motor block. This condition may be a motor symptom of advanced PD as well as it can constitute the "Primary Progressive Freezing of Gait" (PPFG). Our group demonstrated a normal myocardial sympathetic activity in PPFG differently from patients with freezing and $\mathrm{PD}$ presenting severe reduction of $m \mathrm{IBG}$ uptake [51]

\section{Limits in sensitivity and specificity}

Possible causes of false positive and false negative results of mIBG should be kept in mind. It should be noted that a decreased myocardial $m I B G$ uptake is not specific of $L B D$ and PD; in fact, various heart diseases and diabetes may damage the postganglionic sympathetic neurons, leading to false-positive $m$ IBG findings [52, 53]. In particular false-positive results may also be age-related and non-specific for postganglionic sympathetic degeneration, showing myocardial mIBG uptake a significant age-related decrease.

False-negative results of $m \mathrm{IBG}$ scintigraphy in patients with $\mathrm{PD}$ may be caused by an early stage of disease, in presence of a disease duration less than one year, by tremor-dominant phenotypes, by some genetically determined PD [54].

\section{Conclusion}

Metaiodobenzylguanidine was developed initially as a tracer for oncological imaging $[55,56]$; when labeled with ${ }^{123}$ or ${ }^{131}$, it may detect apudomas, such as pheochromocytomas and paragangliomas [57]. In the last years mIBG has found an important role also in neurology and cardiology, as cardiac innervation's tracer [58]. Myocardial $m I B G$ scintigraphy helps to differentiate between PD and other parkinsonian syndromes in clinically difficult cases. Furthermore, the myocardial $\mathrm{mIBG}$ scintigraphy allows insights into the pathophysiology of PD, significantly correlating with the motor phenotype and with the nigrostriatal function (measured by DATscan). These facts suggest that cerebral nigrostriatal dopaminergic degeneration and myocardial sympathetic degeneration coexist in PD. However new emerging data support the idea that dopamine and sympathetic involvement may not progress linearly in parallel; sometimes mIBG's cardiac uptake reduction precedes motor impairment and dopamine degeneration in $\mathrm{PD}$ patients. Finally the contemporary use of MIBG and DATscan permits to distinguish specific pathological entities with similar symptoms.

\section{References}

1. Bengel FM, Schwaiger M. Assessment of cardiac sympathetic neuronal function using PET imaging. J Nucl Cardiol 2004; 11: 603-616.

2. Sisson JC, Wieland DM. Radiolabeled meta-iodobenzylguanidine: Pharmacology and clinical studies. Am J Physiol Imaging 1986; 1: 96-103.

3. Somsen GA, Verberne HJ, Fleury E, Righetti A. Normal values and within-subject variability of cardiac I-123 MIBG scintigraphy in healthy individuals: implications for clinical studies. J Nucl Cardiol 2004; 11: 126-133.

4. Jacobson AF, Senior R, Cerqueira MD et al. ADMIRE-HF Investigators. Myocardial iodine-123 meta-iodobenzylguanidine imaging and cardiac events in heart failure. Results of the prospective ADMIRE-HF (AdreView Myocardial Imaging for Risk Evaluation in Heart Failure) study. J Am Coll Cardiol 2010; 55: 2212-2221.

5. Patel AD, Iskandrian AE. MIBG imaging. J Nucl Cardiol 2002; 9: 75-94.

6. Agostini D, Carrio I, Verberne HJ. How to use myocardial 123I-MIBG scintigraphy in chronic heart failure. Eur J Nucl Med Mol Imaging 2009; 36: 555-559

7. Goldstein DS, Holmes C, Cannon RO 3rd, Eisenhofer G, Kopin IJ. Sympathetic cardioneuropathy in dysautonomias. N Engl J Med 1997; 336: 696-702.

8. Amino T, Orimo S, Itoh Y, Takahashi A, Uchihara T, Mizusawa H. Profound cardiac sympathetic denervation occurs in Parkinson disease. Brain Pathol 2005; 15: 29-34 
9. Orimo S, Takahashi A, Uchihara T, Mori F, Kakita A, Wakabayashi K, Takahashi $\mathrm{H}$. Degeneration of cardiac sympathetic nerve begins in the early disease process of Parkinson"s disease. Brain Pathol 2007; 17: 24-30.

10. Arabia G, Novellino F, Morelli M et al. Mixed tremors with integrity of nigrostriatal system: a clinical and DAT-SPECT follow-up study. Mov Disord 2010; 25: 662-664.

11. Novellino F, Arabia G, Bagnato A et al. Combined use of DAT-SPECT and cardiac MIBG scintigraphy in mixed tremors. Mov Disord 2009 15; 24: 2242-2248.

12. Steele JC, Richardson JC, Olszewski J. Progressive supranuclear palsy. Arch Neurol 1964; 10: 333-359.

13. Rebeiz JJ, Kolodny EH, Richardson EP. Corticodentatonigral degeneration with neuronal achromasia. Arch Neurol 1968; 18: 20-33.

14. Boeve BF, Lang AE, Litvan I. Corticobasal degeneration and its relationship to progressive supranuclear palsy and frontotemporal dementia. Ann Neurol 2003; 54: S15-S19

15. Graham JG, Oppenheimer DR. Orthostatic hypotension and nicotine sensitivity in a case of multiple system atrophy. J Neurol Neurosurg Psychiatry 1969; 32: 28-34

16. Gilman S, Wenning GK, Low PA. Second consensus statement on the diagnosis of multiple system atrophy. Neurology 2008; 71: 670-676.

17. Goldstein DS, Sewell L, Holmes C. Orthostatic hypotension from sympathetic denervation in Parkinson's disease. Neurology 2002; 58: 1247-1255.

18. Goldstein DS, Sewell L, Holmes C. Association of anosmia with autonomic failure in Parkinson disease. Neurology 2010; 74: 245-251.

19. Kashihara K, Imamura T, Shinya T. Cardiac 123I-MIBG uptake is reduced more markedly in patients with REM sleep behavior disorder than in those with early stage Parkinson's disease. Parkinsonism Relat Disord 2010; 16: 252-255.

20. Goldstein DS, Holmes C, Bentho O et al. Biomarkers to detect central dopamine deficiency and distinguish Parkinson disease from multiple system atrophy. Parkinsonism Relat Disord 2008; 14: 600-607.

21. Paglionico S, Labate A, Salsone M et al. Involvement of cardiac sympathetic nerve endings in a patient with idiopathic RBD and intact nigrostriatal pathway. Parkinsonism Relat Disord 2009; 15: 789-791

22. Miyamoto T, Miyamoto M, Inoue Y, Usui Y, Suzuki K, Hirata K. Reduced cardiac 123I-MIBGscintigraphy in idiopathic REM sleep behavior disorder . Neurology 2006; 67: 2236-2238

23. Yoshita M, Taki J, Yokoyama K et al. Value of 123I-MIBG radioactivity in the differential diagnosis of DLB from AD. Neurology 2006; 66: 1850-1854.

24. Novellino F, Bagnato A, Salsone M et al. Myocardial (123)I-MIBG scintigraphy for differentiation of Lewy bodies disease from FTD. Neurobiol Aging 2010; 31: 1903-1911.

25. Goldstein DS, Sewell L, Sharabi Y. Autonomic dysfunction in PD: a window to early detection? J Neurol Sci. 2011; 310: 118-122.

26. Morelli M, Arabia G, Salsone M et al. Accuracy of magnetic resonance parkinsonism index for differentiation of progressive supranuclear palsy from probable or possible Parkinson disease. Mov Disord 2011; 26: 527-533.

27. Cerasa A, Morelli M, Augimeri A et al. Prefrontal thickening in PD with levodopa-induced dyskinesias: New evidence from cortical thickness measurement. Parkinsonism Relat Disord 2012 Jun. [Epub ahead of print] PubMed PMID: 22742954.

28. Cerasa A, Pugliese P, Messina D et al. Prefrontal alterations in Parkinson's disease with levodopa-induced dyskinesia during fMRI motor task. Mov Disord 2012; 27: 364-371.

29. Mansi L, Ciarmiello A, Cuccurullo V. PET/MRI and the revolution of the third eye. Eur J Nucl Med Mol Imaging 2012; 39: 1519-1524.

30. Yoshita M. Differentiation of idiopathic Parkinson's disease from striatonigral degeneration and progressive supranuclear palsy using iodine-123 meta-iodobenzylguanidine myocardial scintigraphy. J Neurol Sci 1998; 155: 60-67.

31. Orimo S, Ozawa E, Nakade S, Sugimoto T, Mizusawa H. 123I-meta-iodobenzylguanidine myocardial scintigraphy in Parkinson's disease. J Neurol Neurosurg Psychiatry 1999; 67: 189-194.
32. Watanabe H, leda T, Katayama T, Takeda A, Aiba I, Doyu M. Cardiac 123I-meta-iodobenzylguanidine (MIBG) uptake in dementia with Lewy bodies: comparison with Alzheimer's disease. J Neurol Neurosurg Psychiatry 2001; 70: 781-783.

33. Orimo S, Amino T, Itoh Y, Takahashi A, Kojo T, Uchihara T. Cardiac sympathetic denervation precedes neuronal loss in the sympathetic ganglia in Lewy body disease. Acta Neuropathol 2005; 109: 583-588.

34. Amino T, Orimo S, Itoh Y, Takahashi A, Uchihara T, Mizusawa H. Profound cardiac sympathetic denervation occurs in Parkinson disease. Brain Pathol 2005; 15: 29-34.

35. Nakajima K, Yoshita M, Matsuo S, Taki J, Kinuya S. Iodine-123-MIBG sympathetic imaging in Lewy body diseases and related movement disorders. Q J Nucl Med Mol Imaging 2008; 52: 378-387.

36. Rascol O, Schelosky L. 123I-Metaiodobenzylguanidine scintigraphy in Parkinson's disease and related disorders. Mov Disord 2009; 24: 732-741.

37. Treglia G, Cason E. Diagnostic performance of myocardial innervation imaging using MIBG scintigraphy in differential diagnosis between dementia with Lewy bodies and other dementias: a systematic review and a meta-analysis. J Neuroimaging 2012; 22: 111-117

38. Hishikawa N, Hashizume Y, Yoshida M, Sobue G. Clinical and neuropathological correlates of Lewy body disease. Acta Neuropathol 2003; 105: 341-350.

39. Jost WH, Del Tredici K, Landvogt C, Braune S. Importance of 123I-metaiodobenzylguanidine scintigraphy/single photon emission computed tomography for diagnosis and differential diagnostics of Parkinson syndromes. Neurodegener Dis 2010; 7: 341-347.

40. Treglia G, Cason E, Gabellini A, Giordano A, Fagioli G. Recent developments in innervation imaging using iodine-123-metaiodobenzylguanidine scintigraphy in Lewy body diseases. Neurol Sci 2010; 31: 417-422.

41. Yamashina S, Yamazaki J Neuronal imaging using SPECT. Eur J Nucl Med Mol Imaging 2007; 34: 939-950.

42. Treglia G, Cason E, Stefanelli A et al. MIBG scintigraphy in differential diagnosis of Parkinsonism: a meta-analysis. Clin Auton Res. 2012; 22: 43-55.

43. Orimo S, Suzuki M, Inaba A, Mizusawa H. 123I-MIBG myocardial scintigraphy for differentiating Parkinson's disease from other neurodegenerative parkinsonism: a systematic review and meta-analysis. Parkinsonism Relat Disord 2012; 18: 494-500.

44. Braune S. The role of cardiac metaiodobenzylguanidine uptake in the differential diagnosis of parkinsonian syndromes. Clin Auton Res 2001; 11: 351-355.

45. Köllensperger M, Seppi K, Liener C, Boesch S, Heute D, Mair KJ. Diffusion weighted imaging best discriminates PD from MSA-P: a comparison with tilt table testing and heart MIBG scintigraphy. Mov Disord 2007; 22: 1771-1776.

46. Fröhlich I, Pilloy W, Vaillant M, Diederich NJ. Myocardial MIBG scintigraphy: a useful clinical tool? A retrospective study in 50 parkinsonian patients. Neurol Sci 2010; 31: 403-406.

47. Nagayama H, Hamamoto M, Ueda M, Nagashima J, Katayama Y. Reliability of MIBG myocardial scintigraphy in the diagnosis of Parkinson's disease. J Neurol Neurosurg Psychiatry 2005; 76: 249-251.

48. Spiegel J, Hellwig D, Farmakis $\mathrm{G}$ et al. Myocardial sympathetic degeneration correlates with clinical phenotype of Parkinson's disease. Mov Disord 2007; 22: 1004-1008

49. Orimo S, Kanazawa T, Nakamura A, Uchihara T, Mori F, Kakita A. Degeneration of cardiac sympathetic nerve can occur in multiple system atrophy. Acta Neuropathol 2007; 113: 81-86

50. King AE, Mintz J, Royall DR. Meta-analysis of (123) I-MIBG cardiac scintigraphy for the diagnosis of Lewy body- related disorders. Mov Disord 2011; 26: 1218-1224

51. Salsone M, Bagnato A, Novellino F et al. Cardiac MIBG scintigraphy in primary progressive freezing gait. Parkinsonism Relat Disord 2009; 15: 365-369.

52. Kyuma M, Nakata T, Hashimoto A, Nagao K, Sasao H, Takahashi T. Incremental prognostic implications of brain natriuretic peptide, cardiac 
sympathetic nerve innervation, and non cardiac disorders in patients with heart failure. J Nucl Med 2004; 45: 155-163.

53. Gazzaruso C, Coppola A, Montalcini T et al. Lipoprotein(a) and homocysteine as genetic risk factors for vascular and neuropathic diabetic foot in type 2 diabetes mellitus. Endocrine 2012; 41: 89-95.

54. Quattrone A, Bagnato A, Annesi G et al. Myocardial 123metaiodobenzylguanidine uptake in genetic Parkinson's disease. Mov Disord 2008; 23: 21-27.

55. Cuccurullo V, Cascini G, Rossi A, Tamburrini O, Rotondo A, Mansi L. Pathophysiological premises to radiotracers for bone metastases. Q J Nucl Med Mol Imaging 2011; 55: 353-373
56. Mansi L, Moncayo R, Cuccurullo V, Dottorini ME, Rambaldi PF. Nuclear medicine in diagnosis, staging and follow-up of thyroid cancer. Q J Nucl Med Mol Imaging 2004; 48: 82-95.

57. Cuccurullo V, Mansi L. Toward tailored medicine (and beyond): the phaeochromocytoma and paraganglioma model. Eur J Nucl Med Mol Imaging 2012; 39: 1262-1265.

58. Teresińska A. Metaiodobenzylguanidine scintigraphy of cardiac sympathetic innervations. Nuclear Medicine Review 2012; 15: 61-70. 